\title{
SUR TROIS NOUVEAUX AMPHIPODES MÉDITERRANÉENS APPARTENANT AU GENRE COROPHIUM LATREILLE
}

PAR

\section{Ed. CHEVREUX}

On connait, en Méditerranée, trois espèces du genre Corophium : C. acherusicum Costa, le plus commun de tous, très répandu sur le littoral français et sur les côtes d'Algérie et de

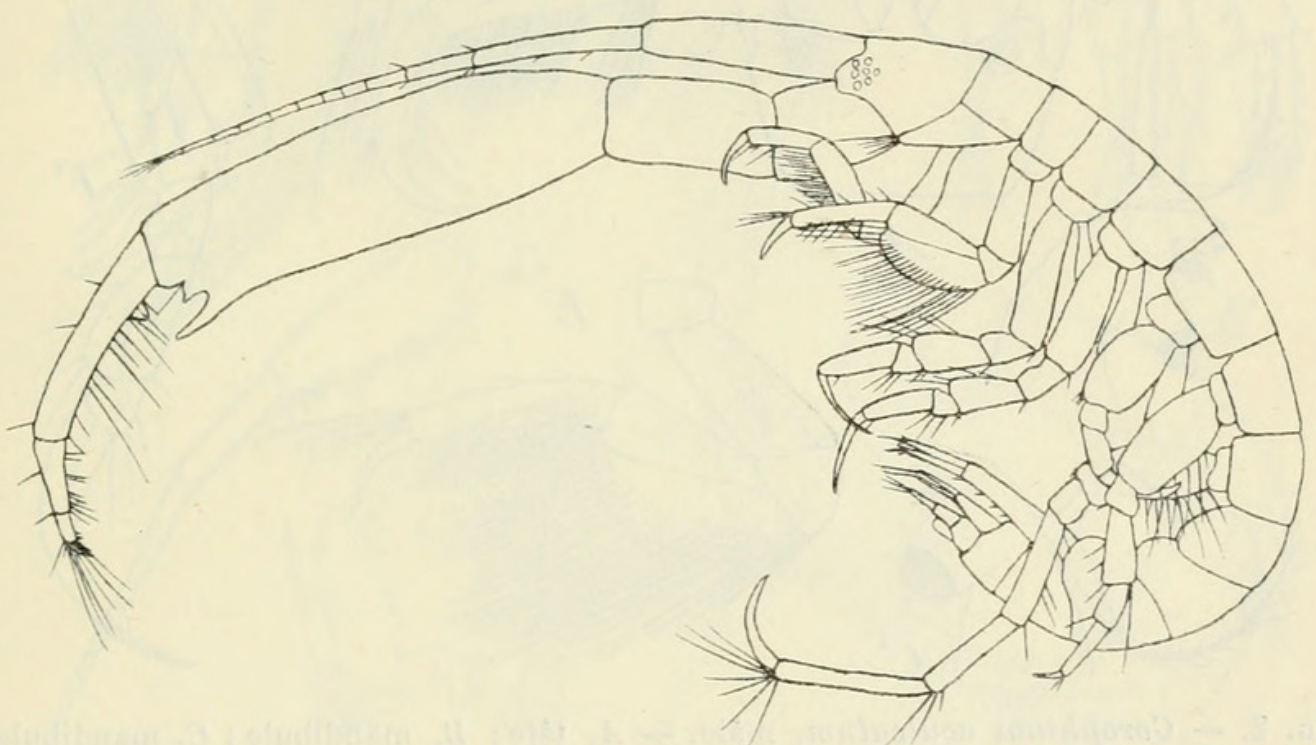

Fig. 1. - Corophium aculeatum. - Nâle, vu du còté gauche, $\times 28$.

Tunisie; C. runcicorne Della Valle, décrit d'après des exemplaires de Naples, retrouvé par Sowinsky dans le Bosphore et dragué à Bonifacio et à Alger par la "Melita "; C. volutator (Pallas), signalé dans l'Adriatique et à Antibes, et dont je possède des exemplaires provenant de Sicile, de Djidjelli, de Porto Vecchio et de Cannes. Ces trois espèces existent aussi sur les côtes de l'Océan.

Quelques recherches effectuées dans le port de Bône me permettent d'ajouter trois espèces nouvelles à l'énumération pré-: cédente. On trouve, dans la partie du port désignée sous le. nom de grande darse, quatre formes différentes de Corophium. C. acherusicum est commun dans les Algues fixées sur les 
bouées, les deux premières des espèces dont la description suit, $C$. aculeatum et $C$. annulatum, habitent les fonds de vase molle grise de la darse, par une profondeur d'environ 8 mètres, et la dernière espèce, $C$. acutum, dont je n'ai encore rencontré que des femelles, se trouve sur les Éponges et les Ascidies qui garnissent les quais, un peu au-dessous du niveau de la mer.

\section{Gorophium aculeatum, nov. sp.}

Mále. - Corps assez grèle, d’un gris rosé translucide, annelé

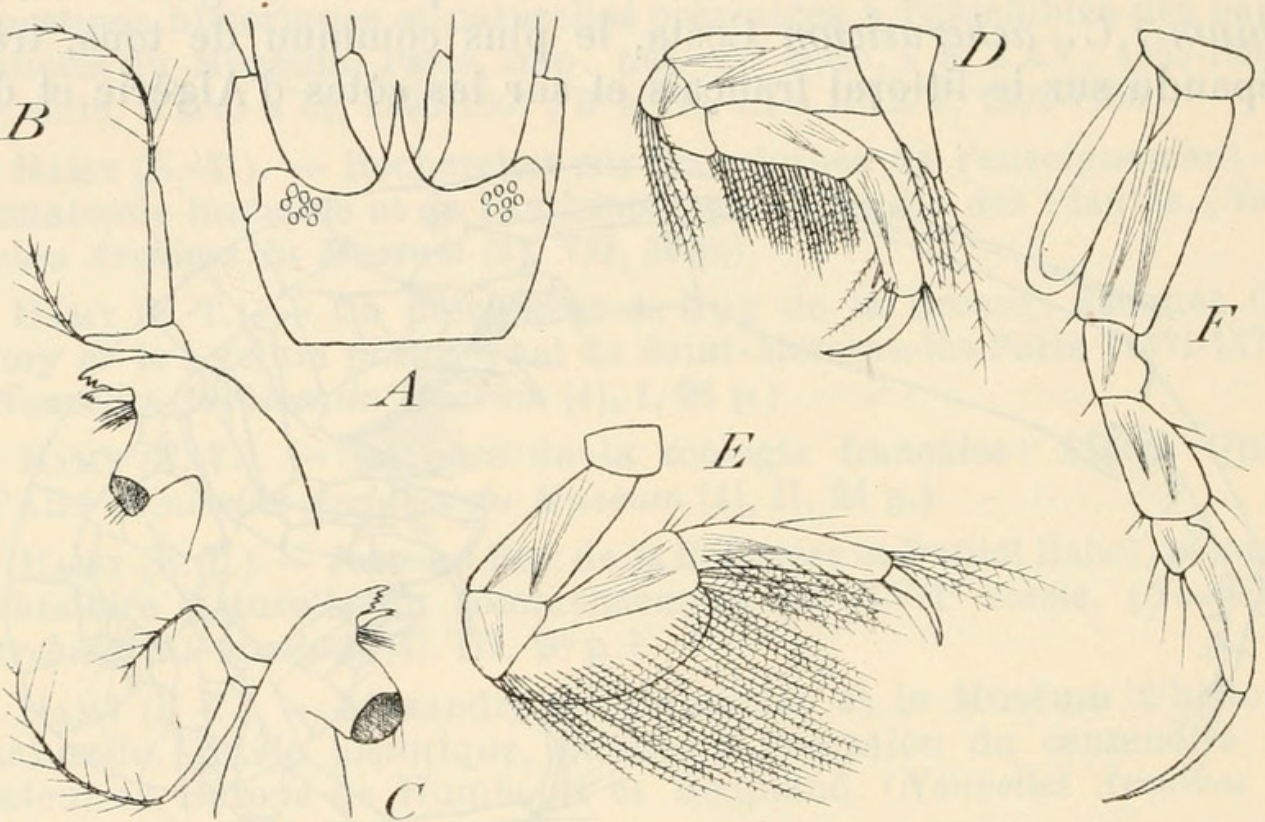

Fıg. 2. - Corophium aculeatum, mảle. - $A$, tète; $B$, mandibule; $C$, mandibule d'un autre exemplaire: $D$, gnathopode antérieur ; $E$, gnathopode postérieur ; $F$, péréiopode de la deuxième paire. $(A \times 32 ; B, C \times 76 ; D, E, F \times 56)$.

de brun violacé dans sa partie dorsale, mesurant un peu moins de 2 millimètres de longueur, dans la position où il est figuré ici (fig. 1). Segments de l'urosome séparés. Tête armée d'un rostre très allongé, grêle et aigu (fig. $2 \mathrm{~A}$ ). Lobes latéraux peu saillants, tronqués à l'extrémité. Yeux comprenant de six à sept ocelles.

Antennes supérieures aussi longues que l'ensemble de la tête et du mésosome. Premier article du pédoncule très allongé, atteignant le double de la longueur de la tête, aussi long que l'ensemble des deux articles suivants. Flagellum aussi long que le premier article du pédoncule et comprenant de neuf à onze articles. Antennes inférieures extraordinairement déve- 
loppées, beaucoup plus longues que le corps. Avant-dernier article du pédoncule très robuste et très allongé, bord postérieur prolongé pour former une dent longue et aiguë, accompagnée d'une dent plus petite. Dernier article du pédoncule court et grêle, portant au bord postérieur une dent triangulaire, qui peut se croiser avec les dents de l'article précédent. Flagellum triarticulé, atteignant la moitié de la longueur du dernier article du pédoncule.

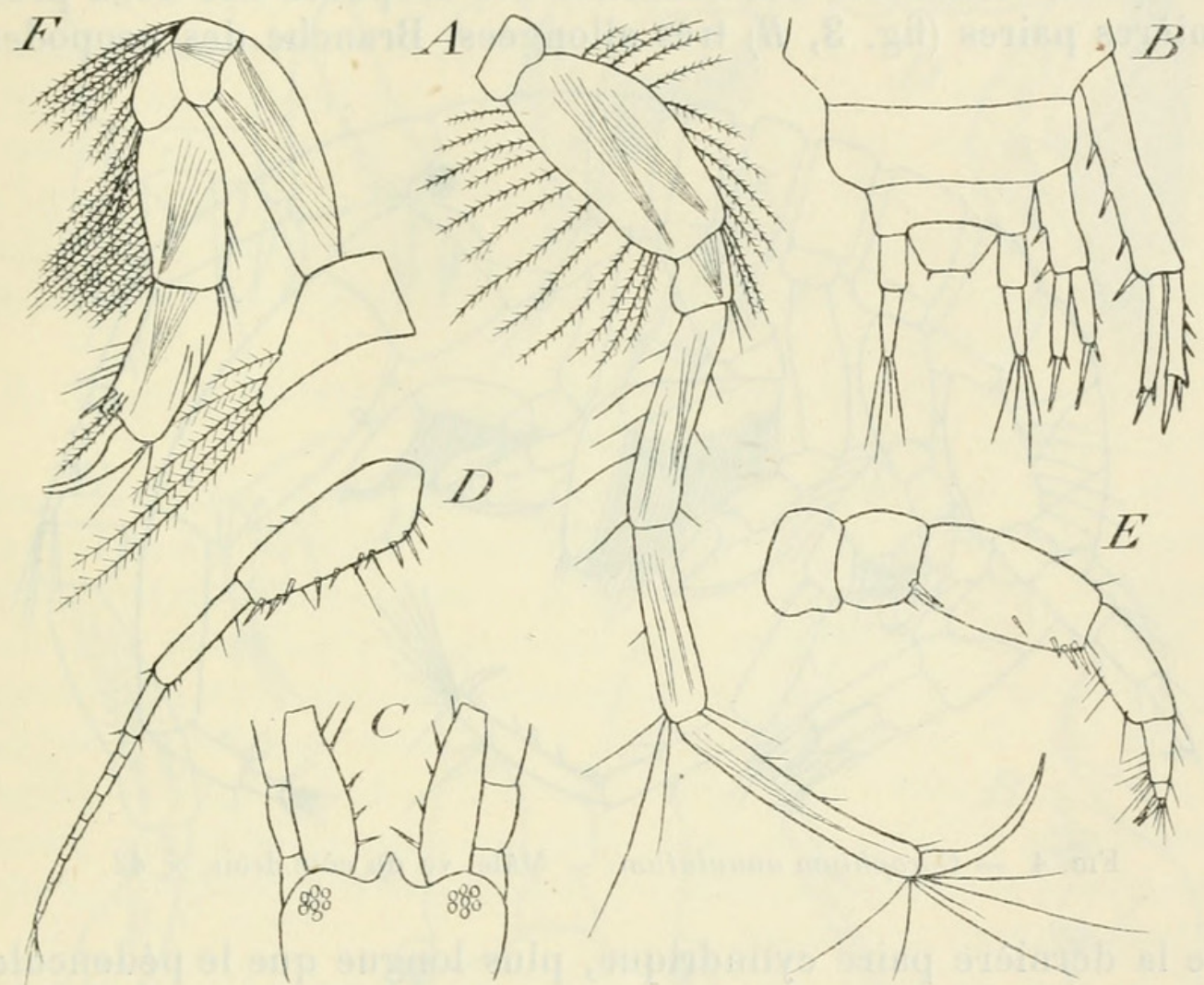

F1G. 3. - Corophium aculeatum. - A, péréiopode de la dernière paire; $B$, urosome du mâle. $-C$, tète; $D$, antenne supérieure; $E$, antenne inférieure; $F$, gnathopode antérieur de la femelle. $(A, B, F \times 70 ; C, D, E \times 32)$.

Palpe des mandibules (fig. 2, $B$ et $C$ ) très remarquable par le prolongement dentiforme de son premier article, qui se terfnine, comme le second article, par une longue soie ciliée.

Propode des gnathopodes antérieurs (fig. 2, D) un peu plus court que le carpe, à peine subchéliforme, son bord palmaire étant extrèmement petit. Dactyle très allongé, presque aussi long que le propode. Article méral des gnathopodes postérieurs (fig. 2, E) atteignant au niveau de l'extrémité du carpe. Propode un peu plus long que le carpe. Dactyle n'atteignant pas la moitié de la longueur du propode. 
Propode des péréiopodes des deux premières paires (fig. 2, $F$ ) un peu plus long que l'article méral. Dactyle atteignant la longueur de l'ensemble du carpe et du propode. Péréiopodes de la dernière paire (fig. $3, A$ ) grêles et très allongés, article basal un peu plus de deux fois aussi long que large, article méral et carpe d'égale longueur, propode beaucoup plus allongé, dactyle remarquablement grand, atteignant près des deux tiers de la longueur du propode.

Épines terminales des branches des uropodes des deux premières paires (fig. $3, B$ ) très allongées. Branche des uropodes

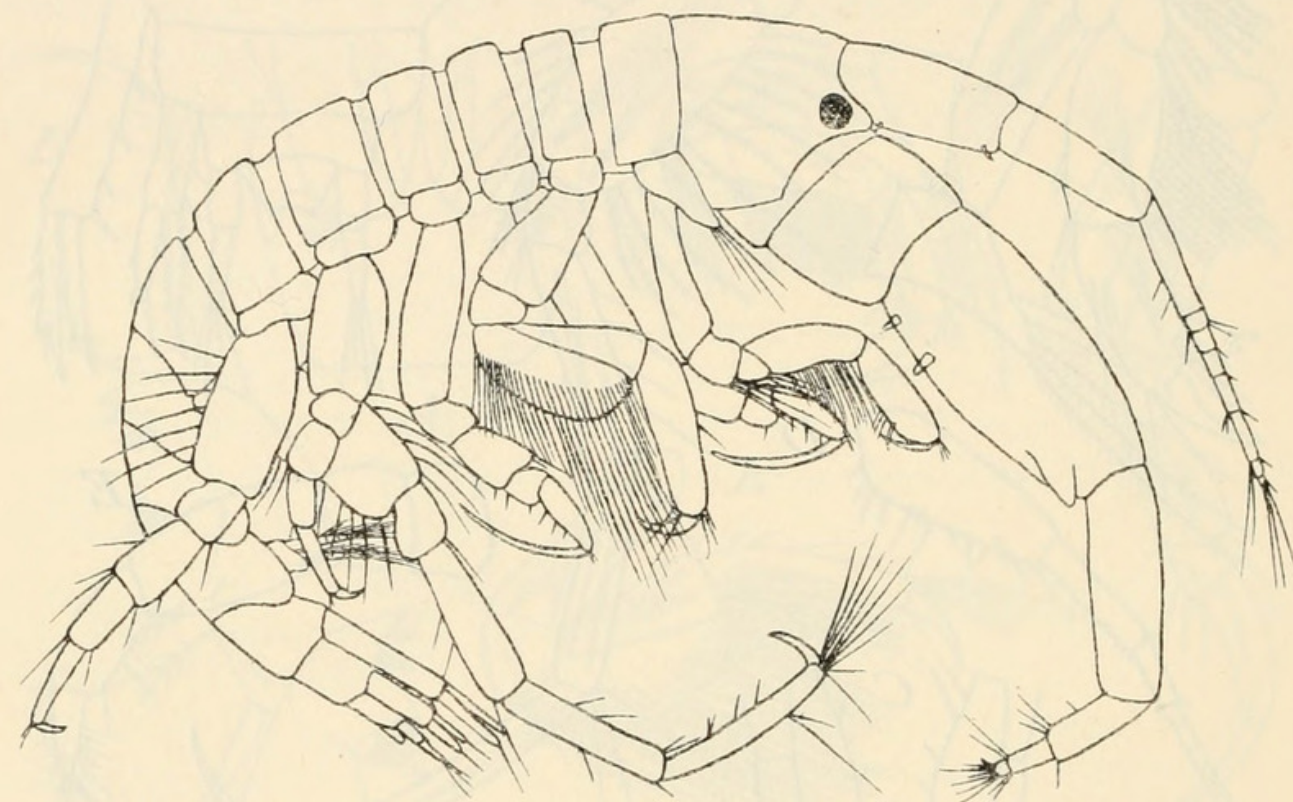

FIg. 4. - Corophium annulatum. - Mâle, vu du côté droit, $\times 42$.

de la dernière paire cylindrique, plus longue que le pédoncule et ne portant que quatre soies terminales. Telson (fig. 3, B) plus large que long, tronqué à l'extrémité, bord distal armé de deux petites épines.

Femelle ovigère. - Un peu plus longue que le màle. Tète (fig. 3, C) armée d'un rostre aigu, beaucoup plus court que celui du mâle. Antennes supérieures (fig. 3, D) moins allongées que celles du mâle, premier article du pédoncule armé de nombreuses épines. Antennes inférieures (fig. $3, E$ ) plus courtes que les antennes supérieures. Pédoncule très robuste, troisième et quatrième articles armés d'épines, mais ne portant pas de dents. Gnathopodes antérieurs (fig. 3, $F$ ) un peu plus robustes que ceux du mâle, dactyle moins allongé.

Le nom spécifique fait allusion à la forme du rostre. 


\section{Gorophium annulatum, nov. sp.}

Mále. - Corps beaucoup plus robuste que celui de l'espèce précédente, coloré en jaune finement ponctué de brun, mesurant $1 \mathrm{~mm}$. 1/2 de longueur dans la position oì il est
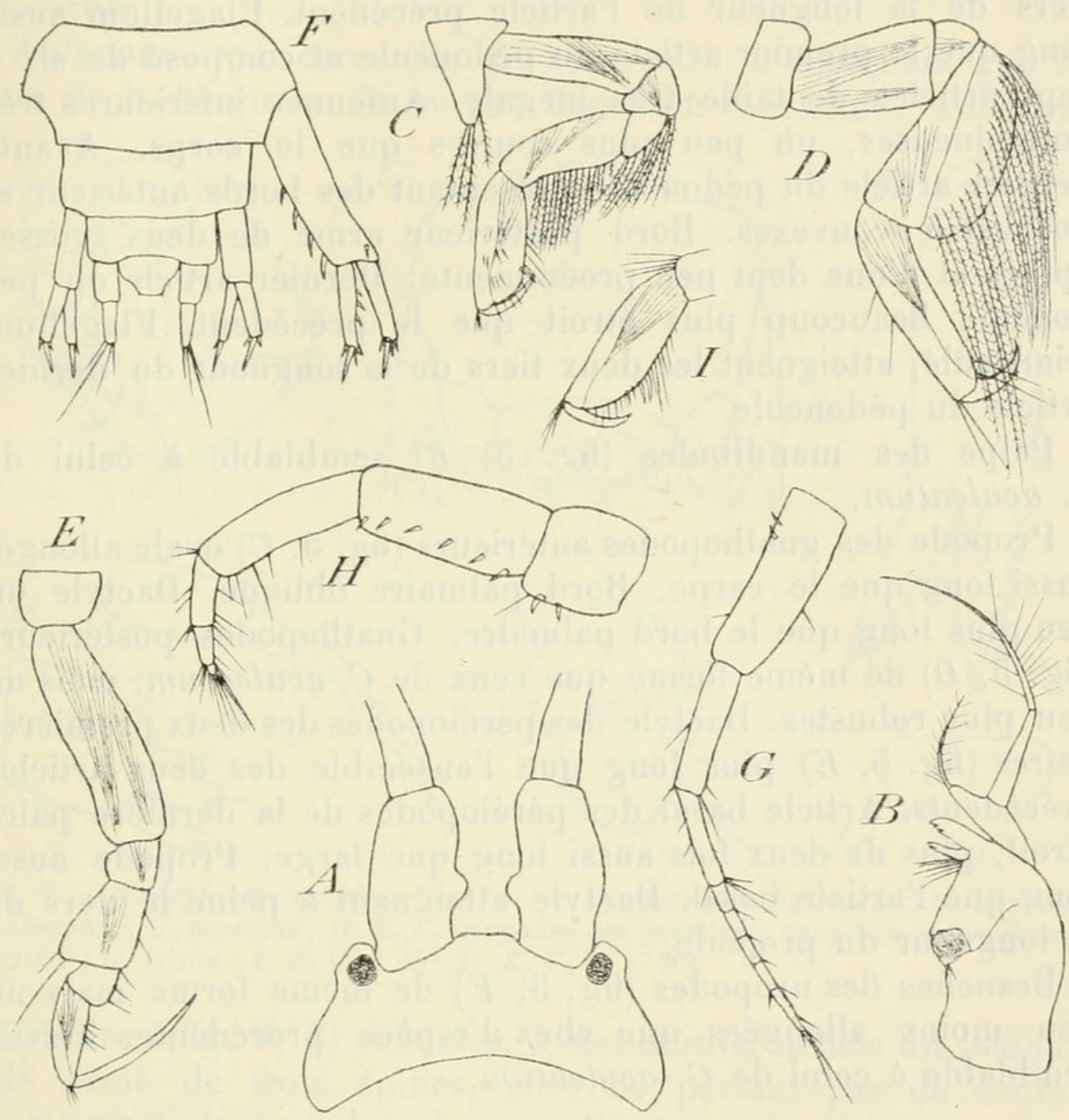

Fig. 5. - Corophizm annulatum. - A, tète; $B$, mandibule; $C$, gnathopode antérieur; $D$, gnathopode postérieur; $E$, péréiopode de la première paire; $F$, urosome du mále. - $G$, antenne supérieure; $H$, antenne inférieure; $I$, extrémité d'un gnathopode antérieur de la femelle. $(A, G, H \times 40 ; B \times 96 ; C, D, E, F, I \times 56)$.

figuré ici (fig. 4). Segments du mésosome renflés, formant une suite d'anneaux nettement séparés les uns des autres. Métasome lisse. Segments de l'urosome séparés. Tète (fig. 5, A) un peu prolongée en avant, mais ne présentant pas de rostre. Lobes latéraux assez saillants, arrondis au bord distal. Yeux ronds et noirs. 
Antennes supérieures aussi longues que l'ensemble de la tête et des six premiers segments du mésosome. Premier article du pédoncule très robuste, à peine plus long que la tête, portant un tubercule au bord interne et une petite épine crochue à chaque extrémité du bord postérieur. Deuxième article aussi long que la tête. Troisième article atteignant les deux tiers de la longueur de l'article précédent. Flagellum aussi long que le premier article du pédoncule et composé de six à sept articles de taille très inégale. Antennes inférieures très volumineuses, un peu plus courtes que le corps. Avantdernier article du pédoncule présentant des bords antérieur et postérieur convexes. Bord postérieur armé de deux grosses épines et d'une dent peu proéminente. Dernier article du pédoncule beaucoup plus étroit que le précédent. Flagellum triarticulé, atteignant les deux tiers de la longueur du dernier article du pédoncule.

Palpe des mandibules (fig. $\ddot{5}, \boldsymbol{B}$ ) semblable à celui de C. aculeatum.

Propode des gnathopodes antérieurs (fig. $5, C$ ) ovale allongé, aussi long que le carpe. Bord palmaire oblique. Dactyle un peu plus long que le bord palmaire. Gnathopodes postérieurs (fig. $\breve{5}, D$ ) de mème forme que ceux de $C$. aculeatum, mais un peu plus robustes. Dactyle des péréiopodes des deux premières paires (fig. 5, E) plus long que l'ensemble des deux articles précédents. Article basal des péréiopodes de la dernière paire étroit, plus de deux fois aussi long que large. Propode aussi long que l'article basal. Dactyle atteignant à peine le tiers de la longueur du propode.

Branches des uropodes (fig. $5, F$ ) de mème forme mais un peu moins allongées que chez l'espèce précédente. Telson semblable à celui de $C$. aculeatum.

Femelle ovigère. - Mésosome moins distinctement annelé que celui du mâle. Antennes supérieures (fig. $b, G$ ) de mème forme mais un peu plus grêles que celles du mâle. Antennes inférieures (fig. $\ddot{3}, H$ ) un peu plus courtes que les antennes supérieures et modérément robustes. Troisième article du pédoncule portant deux épines au bord postérieur. Quatrième article armé de six épines. Cinquième article presque aussi long que l'article précédent. 
Gorophium acutum, nov. sp. (1)

Femelle ovigère. - Corps jaune, avec une tache d'un brun violacé sur la tête et une grande tache de mème couleur sur les plaques coxales, mesurant 3 millimètres de longueur. Eufs d'un jaune clair. Yeux noirs. Segments de l'urosome soudés ensemble.

Antennes supérieures (fig. 6, A) courtes, n'atteignant que le tiers de la longueur du corps; flagellum 6-articulé. Antennes
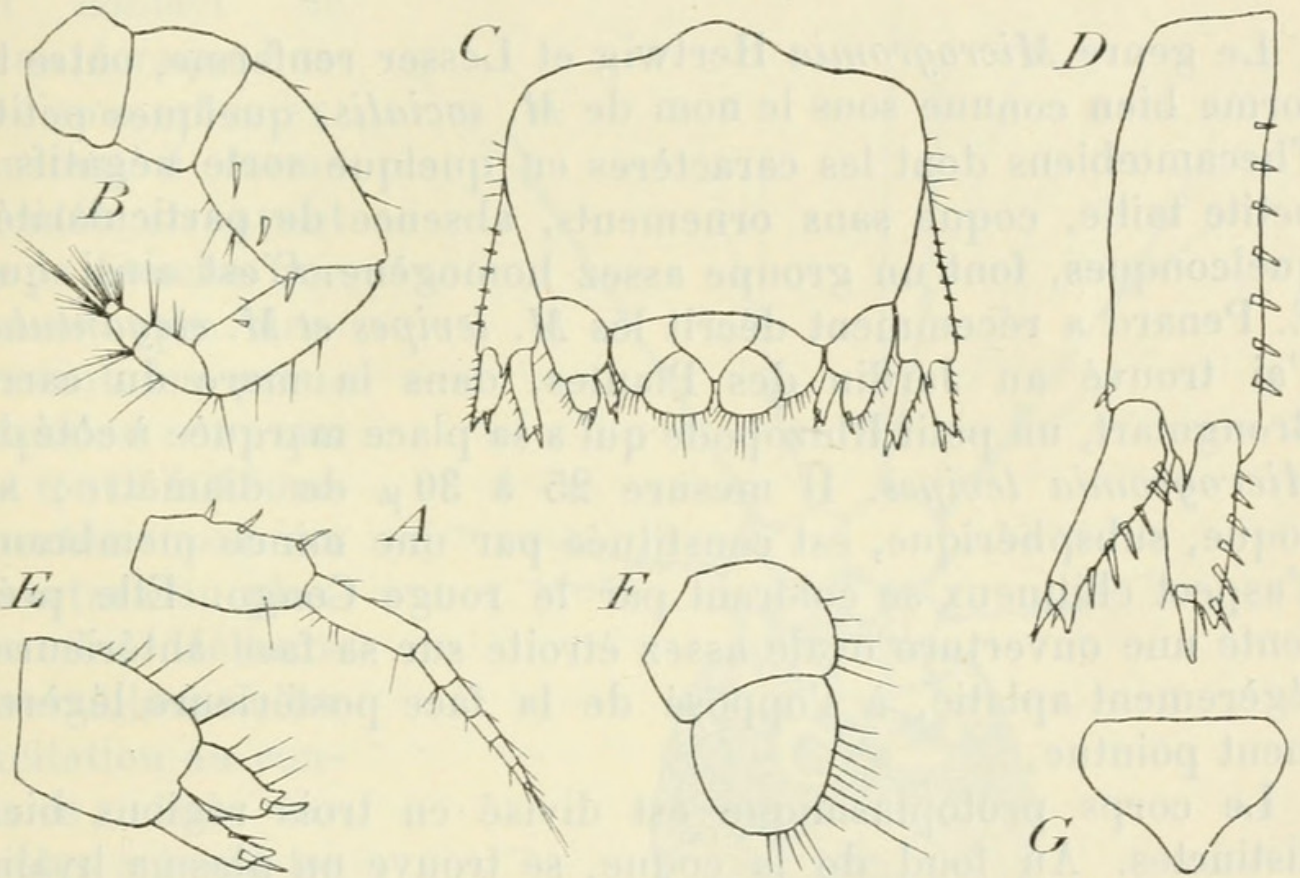

FIg. 6. - Corophium acutum, femelle. - $A$, antenne supérieure; $B$, antenne inférieure; $C$, urosome; $D, E, F$, uropodes des première, deuxième et troisième paires; $G$, telson $(A, B, C \times 40 ; D, E, F, G \times 96)$.

inférieures (fig. $6, B$ ) robustes, avant-dernier article du pédoncule armé de trois épines, mais ne portant pas de dents. Premier article du palpe des mandibules présentant un court prolongement dentiforme, terminé par une soie ciliée. Gnathopodes et péréiopodes différant à peine de ceux de $C$. acherusicum. Uropodes (fig. 6, C) très courts. Branche des uropodes de la dernière paire (fig. $6, F$ ) arrondie, aussi large que longue. Telson (fig, $6, G$ ) subtriangulaire, rétréci dans sa partie distale et terminé en pointe.

Le nom spécifique fait allusion à la forme du telson.

(1) N'ayant encore rencontré que des femelles de cette espèce, j'en donne seulement une courte diagnose, me réservant de la décrire plus completement lorsque j'aurai trouvé le mâle. 


\section{$2 \mathrm{BHL}$ Biodiversity Heritage Library}

Chevreux, Édouard. 1908. "Sur trois nouveaux amphipodes Mediterraneens appartenant au genre Corophium Latreille." Bulletin de la Société zoologique de France 33, 69-75. https://doi.org/10.5962/bhl.part.19156.

View This Item Online: https://www.biodiversitylibrary.org/item/18804

DOI: https://doi.org/10.5962/bhl.part.19156

Permalink: https://www.biodiversitylibrary.org/partpdf/19156

\section{Holding Institution}

MBLWHOI Library

\section{Sponsored by}

MBLWHOI Library

\section{Copyright \& Reuse}

Copyright Status: NOT_IN_COPYRIGHT

This document was created from content at the Biodiversity Heritage Library, the world's largest open access digital library for biodiversity literature and archives. Visit BHL at https://www.biodiversitylibrary.org. 